\title{
Atomic Local Ordering in amorphous InGaZnO
}

Takahiro Suzuki ${ }^{1,2}$ and Kunio Takayanagi, ${ }^{1,2}$

1. Tokyo Institute of Technology, 2-12-1-H-51, Oh-okayama, Meguro, Tokyo 152-8551, JAPAN

2. JST, CREST, 2-12-1-H-51, Oh-okayama, Meguro, Tokyo 152-8551, JAPAN

Amorphous materials are important because of their unique physical properties as demonstrated by aSilicon, a-SiO2, metallic glass, amorphous oxide semiconductors. Transparent amorphous oxide semiconductors (TAOSs) such as amorphous InGaZnO (a-IGZO) have been receiving a good deal of attention [1,2]. The overlap between metal s-orbitals causes high conductivity of TAOSs, where metals locate at oxygen octahedral sites. Their electrical and optical properties depend on the electronic structure, which is governed by medium- and short-range order of atomic/ions position. Structure analyses of amorphous have been done chiefly by extended X-ray absorption fine structure (EXAFS) [1] , although X-ray diffraction [3] and high-resolution transmission electron microscopy (HRTEM) [4] are used. EXAFS gives local structures such as bond distances of the nearest and the coordination number which are average of the specimen size. We used an aberration corrected electron microscope, R005, to find metal-metal bonds by use of local autocorrelation (LAC) of the HRTEM images. Here we demonstrate the method applied to a-IGZO.

Transmission electron micrograph (TEM) image of a-IGZO is shown in Fig. 1. Bright spots correspond to image of $\mathrm{In}, \mathrm{Ga}$, or $\mathrm{Zn}$, having the large atomic number. LAC images are obtained from $1 \mathrm{~nm}^{2}$ area in each TEM image. Typical autocorrelation images are shown in Fig. 2. The distance from center to side spot is related to the correlation between the metal atoms. In Fig. 2(b), distance of nearest neighbor is $2.92 \AA$, which corresponding to the metal-metal distance of edge sharing octahedral sites [1].

\section{References:}

[1] K. Nomura, et al., Phys. Rev. B 75, (2007), 035212.

[2] K. Nomura, et al, Nature (London) 488, (2004), 432.

[3] F. M. Machizaud, et al. J. Non-Cryst. Solids 68, (1984) 271.

[4] Y. Hirotsu, et al. Microsc Res Tech. 40, (1988) 284 


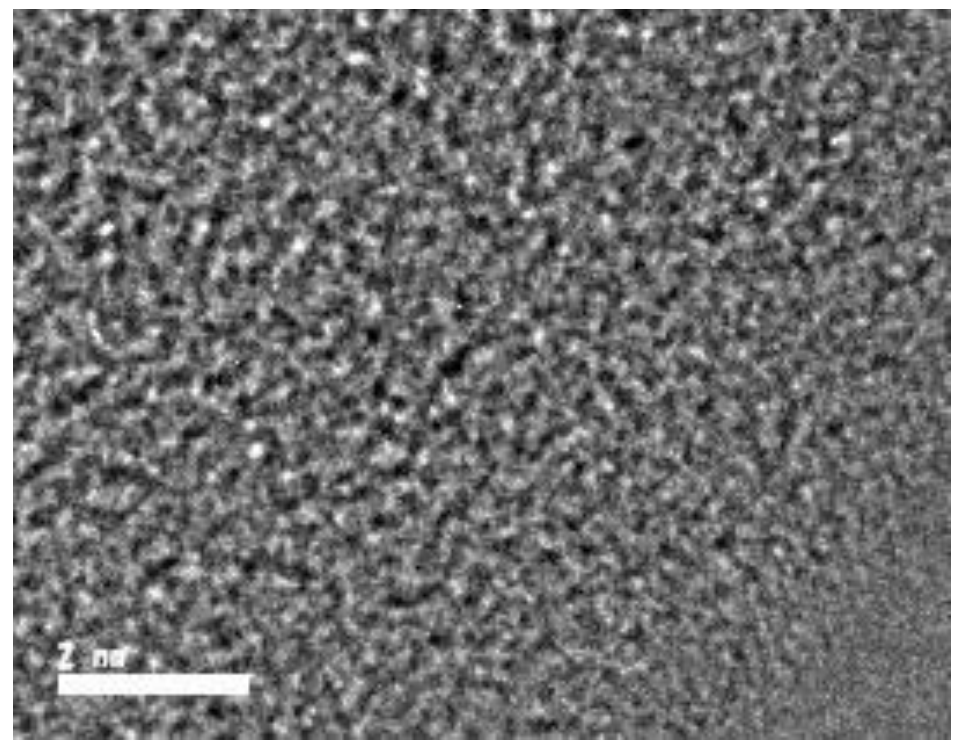

Figure 1. Transmission electron microscope image of amorphous InGaZnO.
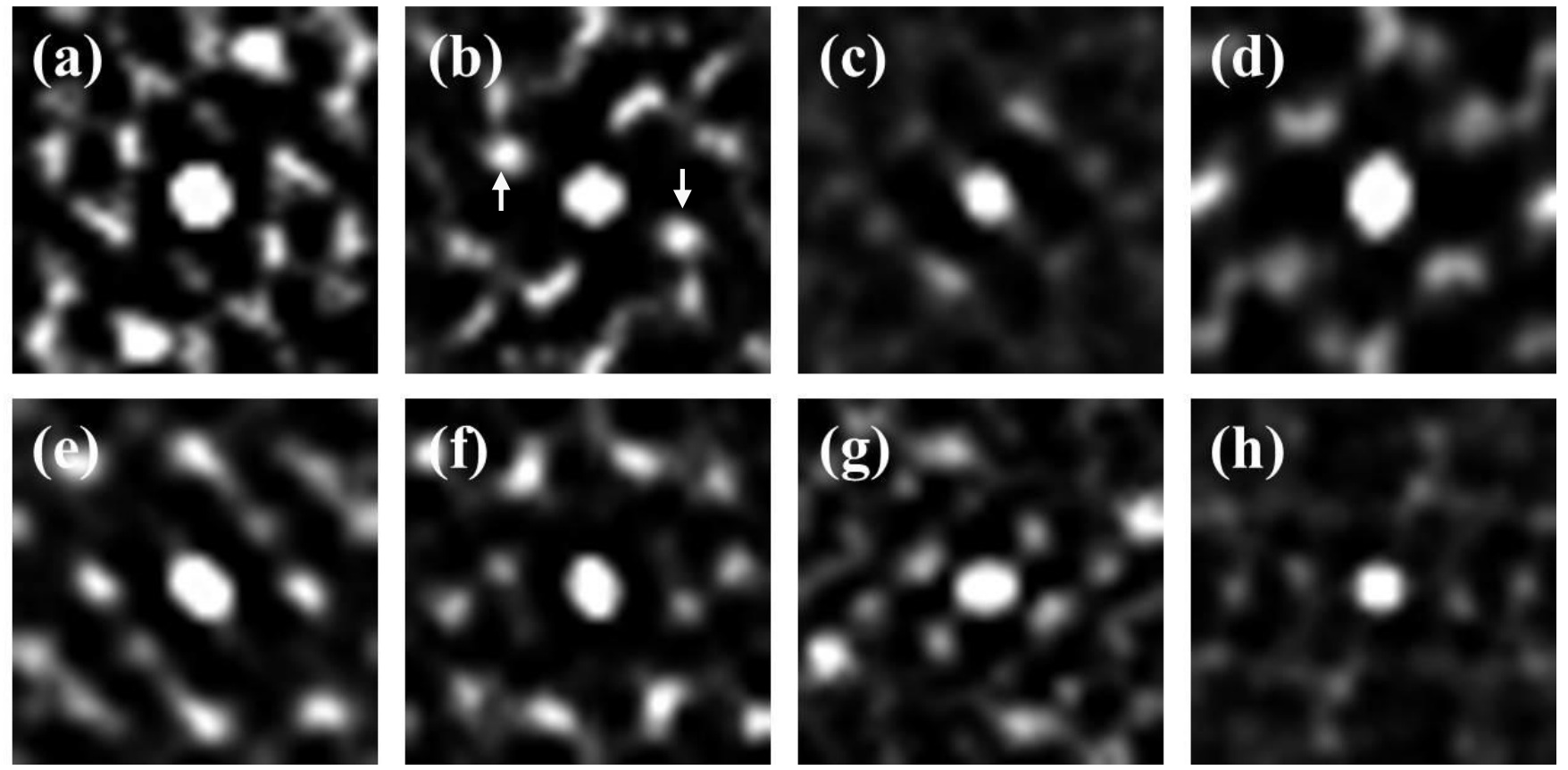

Figure 2. Typical Autocorrelation images. 